Bull. Austral. Math. Soc.

$34 \mathrm{~B} 10,24 \mathrm{~B} 15$

VOL. $71(2005)$ [41-52]

\title{
SOLVABILITY OF SINGULAR SECOND ORDER $m$-POINT BOUNDARY VALUE PROBLEMS OF DIRICHLET TYPE
}

\author{
Ruyun Ma and Bevan Thompson
}

Let $f:[0,1] \times \mathbb{R}^{2} \rightarrow \mathbb{R}$ be a function satisfying the Carathéodory conditions and $t(1-t) e(t) \in L^{1}(0,1)$. Let $a_{i} \in \mathbb{R}$ and $\xi_{i} \in(0,1)$ for $i=1, \ldots, m-2$ where $0<\xi_{1}<\xi_{2}<\cdots<\xi_{m-2}<1$. In this paper we study the existence of $C[0,1]$ solutions for the $m$-point boundary value problem

$$
\begin{aligned}
x^{\prime \prime} & =f\left(t, x(t), x^{\prime}(t)\right)+e(t), \quad 0<t<1 \\
x(0) & =0, \quad x(1)=\sum_{i=1}^{m-2} a_{i} x\left(\xi_{i}\right) .
\end{aligned}
$$

The proof of our main result is based on the Leray-Schauder continuation theorem.

\section{INTRODUCTION}

In [4], Gupta, Ntouyas and Tsamatos considered the problem of proving the existence of a $C^{1}[0,1]$ solution of the $m$-point boundary value problem

$$
\begin{aligned}
& x^{\prime \prime}(t)=f_{1}\left(t, x(t), x^{\prime}(t)\right)+e_{1}(t), \quad 0<t<1 \\
& x(0)=0, \quad x(1)=\sum_{i=1}^{m-2} a_{i} x\left(\xi_{i}\right)
\end{aligned}
$$

where $\xi_{i} \in(0,1)$ for $i=1,2, \ldots, m-2$ satisfies $0<\xi_{1}<\xi_{2}<\cdots<\xi_{m-2}<1$, the $a_{i} \in \mathbb{R}, i=1,2, \ldots, m-2$, have the same sign, $e_{1} \in L^{1}[0,1]$, and $f_{1}:[0,1] \times \mathbb{R}^{2} \rightarrow \mathbb{R}$ satisfies the Carathéodory's conditions as well as a growth condition of the form

$$
\left|f_{1}(t, u, v)\right| \leqslant p_{1}(t)|u|+q_{1}(t)|v|+r_{1}(t)
$$

where $p_{1}, q_{1}, r_{1} \in L^{1}[0,1]$.

This, of course, raises the following natural question: What would happen if $f_{1}$ and $e_{1}$ have a higher order singularity at $t=0$ and $t=1$ ? The results of Gupta, Ntouyas and Tsamatos do not apply to the case $t(1-t) e(t) \in L^{1}[0,1]$.

\section{Received 22nd July, 2004}

The first author gratefully acknowledges support from the NSFC (No. 10271095), GG-110-10736-1003, NWNU-KJCXGC-212, the Foundation of Excellent Young Teachers of the Chinese Education Ministry, and the ARC Centre of Excellence for Mathematics and Statistics of Complex Systems.

Copyright Clearance Centre, Inc. Serial-fee code: 0004-9727/05 \$A2.00+0.00. 
The purpose of this paper is to investigate the existence of $C[0,1]$ solutions for the second order $m$-point boundary value problem

$$
\begin{aligned}
x^{\prime \prime}(t) & =f\left(t, x(t), x^{\prime}(t)\right)+e(t), \quad 0<t<1 \\
x(0) & =0, \quad x(1)=\sum_{i=1}^{m-2} a_{i} x\left(\xi_{i}\right)
\end{aligned}
$$

where $f:[0,1] \times \mathbb{R}^{2} \rightarrow \mathbb{R}$ satisfies the Carathéodory conditions (that is, for each $(x, y)$ $\in \mathbb{R}^{2}$, the function $f(\cdot, x, y)$ is measurable on $[0,1]$ and for almost every $t \in[0,1]$, the function $f(t, \cdot, \cdot)$ is continuous on $\left.\mathbb{R}^{2}\right)$. We make the following additional assumptions:

(H0) $\quad a_{i} \in \mathbb{R}$ and $\xi_{i} \in(0,1)$ for $i=1,2, \ldots, m-2$ where $0<\xi_{1}<\xi_{2}<\ldots$ $<\xi_{m-2}<1$ and

$$
\sum_{i=1}^{m-2} a_{i} \xi_{i} \neq 1
$$

(H1) There exist $q(t) \in L^{1}[0,1]$ and $p(t), r(t) \in L_{\text {loc }}^{1}(0,1)$ with $t(1-t) p(t)$, $t(1-t) r(t) \in L^{1}[0,1]$, such that

$|f(t, u, v)| \leqslant p(t)|u|+q(t)|v|+r(t), \quad$ almost everywhere $t \in[0,1], \quad(u, v) \in \mathbb{R}^{2}$

where

$L_{\text {loc }}^{1}(0,1)=\left\{u|u|_{[c, d]} \in L^{1}[c, d]\right.$ for every compact interval $\left.[c, d] \subset(0,1)\right\}$;

(H2) The function $e:[0,1] \rightarrow \mathbb{R}$ satisfies $\int_{0}^{1} t(1-t)|e(t)| d t<\infty$.

For results concerning the existence and multiplicity of solutions (or positive solutions) of singular nonlinear two-point boundary value problems, one may refer, to Agarwal and O'Regan [1], Asakawa [2], Baxley [3], O'Regan [7], Shi and Chen [8] and Taliaferro [10] and the references therein. The existence and multiplicity of solutions of non-singular multi-point boundary value problems have been studied by many authors; see, for example, Gupta [4], Ma $[4,5]$, Webb [10] and the references therein for more information on this problem. For recent results on singular multi-point boundary value problems, see Zhang and Wang [11].

\section{Preliminary Lemmas}

In this section, we always assume that (HO) holds.

We shall use the classical Banach spaces $C[0,1], C^{k}[0,1], L^{1}[0,1]$ and $L^{\infty}[0,1]$. We denote by $A C[a, b]$ the space of all absolutely continuous functions on $[a, b]$, and define $A C^{k}[a, b]$ by

$$
A C^{k}[a, b]=\left\{u \in C^{k}[a, b] \mid u^{(k)} \in A C[a, b]\right\}
$$


where $A C^{0}[a, b]=A C[a, b]$. Let $I$ be an interval in $R$. We denote by $A C_{\text {loc }} I$ and $L_{\text {loc }}^{1} I$ the spaces of functions on $I$ defined by

$$
A C_{\text {loc }} I=\left\{u|u|_{[c, d]} \in A C[c, d] \text { for every compact interval }[c, d] \subset I\right\}
$$

and

$$
L_{\text {loc }}^{1} I=\left\{u|u|_{\{c, d]} \in L^{1}[c, d] \text { for every compact interval }[c, d] \subset I\right\} .
$$

Let $E$ be the Banach space

$$
E=\left\{y \in L_{\mathrm{loc}}^{\mathrm{l}}(0,1) \mid t(1-t) y(t) \in L^{1}[0,1]\right\}
$$

equipped with the norm

$$
\|y\|_{E}=\int_{0}^{1} t(1-t)|y(t)| d t
$$

and let $X$ be the Banach space

$$
X=\left\{u \in C^{1}(0,1) \mid u \in C[0,1], \lim _{t \rightarrow 1}(1-t) u^{\prime}(t) \text { and } \lim _{t \rightarrow 0} t u^{\prime}(t) \text { exist }\right\}
$$

equipped with the norm

$$
\|u\|_{X}=\max \left\{\|u\|_{\infty},\left\|t(1-t) u^{\prime}(t)\right\|_{\infty}\right\}
$$

where $\|\cdot\|_{\infty}$ denotes the sup norm.

Let $G(t, s)$ be the Green's function for the second-order boundary value problem

$$
\begin{aligned}
-u^{\prime \prime}(t) & =0, \quad t \in(0,1) \\
u(0)=u(1) & =0
\end{aligned}
$$

which is explicitly given by

$$
G(t, s)= \begin{cases}(1-t) s, & 0 \leqslant s \leqslant t \leqslant 1 \\ (1-s) t, & 0 \leqslant t \leqslant s \leqslant 1 .\end{cases}
$$

For each $y \in E$, we define the operator $T$ by

$$
(T y)(t)=\int_{0}^{1} G(t, s) y(s) d s+\frac{t}{1-\sum_{i=1}^{m-2} a_{i} \xi_{i}} \sum_{i=1}^{m-2} a_{i} \int_{0}^{1} G\left(\xi_{i}, s\right) y(s) d s .
$$


Now since

$$
\begin{aligned}
& \left|\int_{0}^{1} G(t, s) y(s) d s+\frac{t}{1-\sum_{i=1}^{m-2} a_{i} \xi_{i}} \sum_{i=1}^{m-2} a_{i} \int_{0}^{1} G\left(\xi_{i}, s\right) y(s) d s\right| \\
& \leqslant \int_{0}^{1} G(t, s)|y(s)| d s+\frac{t}{\left|1-\sum_{i=1}^{m-2} a_{i} \xi_{i}\right|} \sum_{i=1}^{m-2}\left|a_{i}\right| \int_{0}^{1} G\left(\xi_{i}, s\right)|y(s)| d s \\
& \leqslant \int_{0}^{t}(1-t) s|y(s)| d s+\int_{t}^{1}(1-s) t|y(s)| d s \\
& \quad+\frac{1}{\left|1-\sum_{i=1}^{m-2} a_{i} \xi_{i}\right|} \sum_{i=1}^{m-2}\left|a_{i}\right|\left[\int_{0}^{\xi_{i}}\left(1-\xi_{i}\right) s|y(s)| d s+\int_{\xi_{i}}^{1}(1-s) \xi_{i}|y(s)| d s\right] \\
& \leqslant\left(1+\frac{\sum_{i=1}^{m-2}\left|a_{i}\right|}{\left|1-\sum_{i=1}^{m-2} a_{i} \xi_{i}\right|}\right) \int_{0}^{1} s(1-s)|y(s)| d s<\infty
\end{aligned}
$$

we know from $(\mathrm{H} 0)$ that $(T y):[0,1] \rightarrow \mathbb{R}$ is well-defined.

REMARK 2.1. If all of the $a_{i}$ 's have the same sign, then $x(1)=\sum_{i=1}^{m-2} a_{i} x\left(\xi_{i}\right)$ implies

$$
x(1)=\alpha x(\eta)
$$

for some $\eta \in\left[\xi_{1}, \xi_{m-2}\right]$, where $\alpha=\sum_{i=1}^{m-2} a_{i}$. To study (1.4)-(2.5), Gupta, Ntouyas and Tsamatos defined the operator $T$ by

$$
(T y)(t)=\int_{0}^{t}(t-s) y(s) d s+\frac{\alpha t}{1-\alpha \eta} \int_{0}^{\eta}(\eta-s) y(s) d s-\frac{t}{1-\alpha \eta} \int_{0}^{1}(1-s) y(s) d s .
$$

This form of $T$ is not suitable for studying the multi-point boundary value problem (1.4)-(2.5), and accordingly (1.4)-(1.5), in singular case.

Lemma 2.1. ([2, Lemma 2.1].) Suppose that $\phi \in E$. Then

(i) $\int_{0}^{t} s \phi(s) d s, \int_{t}^{1}(1-s) \phi(s) d s \in L^{1}[0,1]$, and

$$
\int_{0}^{1} \int_{0}^{t} s \phi(s) d s d t=\int_{0}^{1} \int_{t}^{1}(1-s) \phi(s) d s d t=\int_{0}^{1} s(1-s) \phi(s) d s
$$

(ii) $\lim _{t \rightarrow 0} t \int_{t}^{1}(1-s) \phi(s) d s=0, \lim _{t \rightarrow 1}(1-t) \int_{0}^{t} s \phi(s) d s=0$.

Lemma 2.2. Let $y \in E$. Then $T y \in X$ and

$$
(T y)^{\prime \prime}(t)+y(t)=0, \quad \text { almost everywhere } t \in(0,1) .
$$


ProOF: For $y(t) \in E$, we have that $t(1-t) y(t) \in L^{1}[0,1]$. So for each $r \in(0,1)$, $t y(t) \in L^{1}[0, r]$ and $(1-t) y(t) \in L^{1}[r, 1]$. Thus $(T y)(t) \in A C_{\mathrm{loc}}(0,1)$ since

$$
\begin{aligned}
(T y)(t)= & \int_{0}^{t}(1-t) s y(s) d s+\int_{t}^{1}(1-s) t y(s) d s \\
& +\frac{t}{1-\sum_{i=1}^{m-2} a_{i} \xi_{i}} \sum_{i=1}^{m-2} a_{i}\left[\int_{0}^{\xi_{i}}\left(1-\xi_{i}\right) s y(s) d s+\int_{\xi_{i}}^{1}(1-s) \xi_{i} y(s) d s\right]
\end{aligned}
$$

Moreover

$$
(T y)^{\prime}(t)=-\int_{0}^{t} s y(s) d s+\int_{t}^{1}(1-s) y(s) d s+D_{y}
$$

where

$$
D_{y}:=\frac{1}{1-\sum_{i=1}^{m-2} a_{i} \xi_{i}} \sum_{i=1}^{m-2} a_{i} \int_{0}^{1} G\left(\xi_{i}, s\right) y(s) d s
$$

Now since

$$
\begin{aligned}
\int_{0}^{1}\left|(T y)^{\prime}(t)\right| d t & =\int_{0}^{1}\left|-\int_{0}^{t} s y(s) d s+\int_{t}^{1}(1-s) y(s) d s+D_{y}\right| d t \\
& \leqslant \int_{0}^{1} \int_{0}^{t} s|y(s)| d s d t+\int_{0}^{1} \int_{t}^{1}(1-s)|y(s)| d s d t+\left|D_{y}\right| \\
& =\int_{0}^{1} \int_{s}^{1} s|y(s)| d t d s+\int_{0}^{1} \int_{0}^{s}(1-s)|y(s)| d t d s+\left|D_{y}\right| \\
& =2 \int_{0}^{1} s(1-s)|y(s)| d s+\left|D_{y}\right|<\infty
\end{aligned}
$$

we have $T y \in A C[0,1]$. Now (2.8) together with the fact that $s y(s) \in L^{1}[0, r]$ and $(1-s) y(s) \in L^{1}[r, 1]$ for each $r \in(0,1)$ imply that $(T y)^{\prime}(t) \in A C_{\text {loc }}(0,1)$, and accordingly

$$
(T y)^{\prime \prime}(t)=-y(t), \quad \text { almost everywhere } t \in(0,1) .
$$

Now set

$$
\gamma(t):=\left[t(1-t)(T y)^{\prime}(t)\right]^{\prime}, \quad t \in[0,1] .
$$

First we show $\gamma \in L^{1}[0,1]$. If this is true then $t(1-t)(T y)^{\prime}(t) \in A C[0,1]$, and consequently, $\lim _{t \rightarrow 1}(1-t)(T y)^{\prime}(t)$ and $\lim _{t \rightarrow 0} t(T y)^{\prime}(t)$ exist. 
A simple computation (by interchanging the order of integration) yields

$$
\begin{aligned}
\int_{0}^{1}|\gamma(t)| d t= & \int_{0}^{1}\left|\left[(1-t)(T y)^{\prime}(t)-t(T y)^{\prime}(t)+t(1-t)(T y)^{\prime \prime}(t)\right]\right| d t \\
\leqslant & \int_{0}^{1} .(1-t)\left|(T y)^{\prime}(t)\right| d t+\int_{0}^{1} t\left|(T y)^{\prime}(t)\right| d t+\int_{0}^{1} t(1-t)\left|(T y)^{\prime \prime}(t)\right| d t \\
\leqslant & \int_{0}^{1}\left[(1-t) \int_{0}^{t} s|y(s)| d s\right] d t+\int_{0}^{1}\left[(1-t) \int_{t}^{1}(1-s)|y(s)| d s\right] d t \\
& \quad+\int_{0}^{1}(1-t)\left|D_{y}\right| d t+\int_{0}^{1}\left[t \int_{0}^{t} s|y(s)| d s\right] d t \\
& \quad+\int_{0}^{1}\left[t \int_{t}^{1}(1-s)|y(s)| d s\right] d t+\int_{0}^{1} t\left|D_{y}\right| d t+\int_{0}^{1} t(1-t)|y(t)| d t \\
= & \int_{0}^{1}\left[\int_{s}^{1}(1-t) s|y(s)| d t\right] d s+\int_{0}^{1}\left[\int_{0}^{s}(1-t)(1-s)|y(s)| d t\right] d s \\
& \quad+\int_{0}^{1}\left[\int_{s}^{1} t s|y(s)| d t\right] d s+\int_{0}^{1}\left[\int_{0}^{s} t(1-s)|y(s)| d t\right] d s \\
& \quad+\int_{0}^{1} t(1-t)|y(t)| d t+\left|D_{y}\right| \\
& \int_{0}^{1} s(1-s)|y(s)| d s+\left|D_{y}\right|<\infty .
\end{aligned}
$$

This completes the proof of the lemma.

LEMMA 2.3. Let $y \in E$. Then

$$
(T y)(0)=0 \text { and }(T y)(1)=\sum_{i=1}^{m-2} a_{i}(T y)\left(\xi_{i}\right)
$$

Proof: By Lemma 2.2, $T y \in X$. Thus we have from (2.7) that

$$
\begin{aligned}
(T y)(0)= & \lim _{t \rightarrow 0}(T y)(t) \\
= & \lim _{t \rightarrow 0} \int_{0}^{t}(1-t) s y(s) d s+\lim _{t \rightarrow 0} \int_{t}^{1}(1-s) t y(s) d s \\
& \quad+\frac{0}{1-\sum_{i=1}^{m-2} a_{i} \xi_{i}} \sum_{i=1}^{m-2} a_{i} \int_{0}^{1} G\left(\xi_{i}, s\right) y(s) d s \\
= & 0 .
\end{aligned}
$$

Again applying (2.7) and the fact that $T y \in X$, we have

$$
\begin{aligned}
(T y)(1) & =\lim _{t \rightarrow 1}(T y)(t) \\
& =\lim _{t \rightarrow 1} \int_{0}^{t}(1-t) s y(s) d s+\lim _{t \rightarrow 1} \int_{t}^{1}(1-s) t y(s) d s \\
& \quad+\frac{1}{1-\sum_{i=1}^{m-2} a_{i} \xi_{i}} \sum_{i=1}^{m-2} a_{i} \int_{0}^{1} G\left(\xi_{i}, s\right) y(s) d s .
\end{aligned}
$$


Applying (ii) of Lemma 2.1 and using the fact that $(1-s) y(s) \in L^{1}[r, 1]$ for some $r, 0<r<1$, we conclude that

$$
(T y)(1)=\frac{1}{1-\sum_{i=1}^{m-2} a_{i} \xi_{i}} \sum_{i=1}^{m-2} a_{i} \int_{0}^{1} G\left(\xi_{i}, s\right) y(s) d s
$$

Similarly

$$
(T y)\left(\xi_{i}\right)=\int_{0}^{1} G\left(\xi_{i}, s\right) y(s) d s+\frac{\xi_{i}}{1-\sum_{i=1}^{m-2} a_{i} \xi_{i}} \sum_{i=1}^{m-2} a_{i} \int_{0}^{1} G\left(\xi_{i}, s\right) y(s) d s
$$

This together with (2.13) implies that $(T y)(1)=\sum_{i=1}^{m-2} a_{i}(T y)\left(\xi_{i}\right)$.

For $x \in X$, we define a nonlinear operator $N$ by

$$
(N x)(t)=-f\left(t, x(t), x^{\prime}(t)\right)-e(t), \quad t \in(0,1)
$$

From (H1) and (H2), we conclude that $N: X \rightarrow E$ is well-defined. In fact

$$
\begin{aligned}
\|N x\|_{E} & =\|t(1-t)(N x)(t)\|_{L^{1}} \\
& =\int_{0}^{1} t(1-t)\left|f\left(t, x(t), x^{\prime}(t)\right)+e(t)\right| d t \\
& \leqslant \int_{0}^{1}\left[t(1-t) p(t)|x(t)|+|q(t)| t(1-t) x^{\prime}(t)|+t(1-t)| r(t) \mid\right. \\
& \quad+t(1-t)|e(t)|] d t \\
& \leqslant\|p\|_{E}\|x\|_{\infty}+\|q\|_{L^{1}}\left\|t(1-t) x^{\prime}(t)\right\|_{\infty}+\|r\|_{E}+\|e\|_{E} \\
& <\infty .
\end{aligned}
$$

LEMMA 2.4 TN:X $\rightarrow X$ is completely continuous.

Proof: From the definitions of $T$ and $N$ and (H1) and (H2), it is easy to show that $T N: X \rightarrow X$ is continuous. Let $B \subset X$ be a bounded set. We need to show that $(T N)(B)$ is a relatively compact subset of $X$.

Let $\left\{x_{n}\right\} \subset B$ and set

$$
w_{n}(t)=\left((T N) x_{n}\right)(t) \text { and } z_{n}(t)=t(1-t)\left((T N) x_{n}\right)^{\prime}(t)
$$

We need show only that there exists a subsequence with

$$
w_{n} \rightarrow w^{*} \quad \text { in } C[0,1]
$$

and

$$
z_{n} \rightarrow z^{*} \quad \text { in } C[0,1]
$$


(We note that (2.18) together with (2.19) and the fact that $z^{*}(t)=t(1-t)\left(w^{*}(t)\right)^{\prime}$ for $t \in[0,1)$ implies that, after taking a subsequence if necessary, $\left\|w_{n}-w^{*}\right\|_{X} \rightarrow 0$.)

To prove (2.18), we recall that $N: X \rightarrow E$ and

$$
\begin{aligned}
\left|\left(N x_{n}\right)(t)\right| & \leqslant p(t) M+\frac{q(t)}{t(1-t)} M+r(t)+|e(t)| \\
& :=\chi(t)
\end{aligned}
$$

where

$$
M=\max \left\{\|x\|_{X} \mid x \in B\right\}
$$

Clearly, (H1) and (H2) imply that $\chi \in E$. Now for each $n$ and for every $t_{1}, t_{2} \in[0,1]$ with $t_{1}<t_{2}$

$$
\begin{aligned}
\left|w_{n}\left(t_{1}\right)-w_{n}\left(t_{2}\right)\right| & =\left|\int_{t_{2}}^{t_{1}}\left((T N) x_{n}\right)^{\prime}(\tau) d \tau\right| \\
& \leqslant \int_{t_{2}}^{t_{1}}\left|\left((T N) x_{n}\right)^{\prime}(\tau)\right| d \tau \\
& =\int_{t_{2}}^{t_{1}}\left|-\int_{0}^{\tau} s\left(N x_{n}\right)(s) d s+\int_{\tau}^{1}(1-s)\left(N x_{n}\right)(s) d s+D_{N x_{n}}\right| d \tau \\
& \leqslant \int_{t_{2}}^{t_{1}}\left[\int_{0}^{\tau} s\left|\left(N x_{n}\right)(s)\right| d s+\int_{\tau}^{1}(1-s)\left|\left(N x_{n}\right)(s)\right| d s+D\right] d \tau \\
& =\int_{t_{2}}^{t_{1}}\left[\int_{0}^{\tau} s \chi(s) d s+\int_{\tau}^{1}(1-s) \chi(s) d s+D\right] d \tau
\end{aligned}
$$

where

$$
D=\sup \left\{\left|D_{y}\right| \mid y \in B\right\}
$$

By (i) of Lemma 2.2, $\int_{0}^{\tau} s \chi(s) d s, \int_{\tau}^{1}(1-s)|\chi(s)| d s \in L^{1}[0,1]$. Thus (2.22) shows that $\left\{w_{n}\right\}_{n=1}^{\infty}$ is equi-continuous on $[0,1]$. Therefore by the Arzela-Ascoli theorem, after taking a subsequence if necessary, (2.18) holds.

To prove (2.19), in view of the Arzela-Ascoli theorem, we need to verify that

(a) $\left\|z_{n}\right\|_{\infty}<M_{1}$ for some positive constant $M_{1}$, independent of $n$;

(b) $\left\{z_{n}(t)\right\}_{n=1}^{\infty}$ is equi-continuous on $[0,1]$.

Since (a) can be easily deduced from the definitions of $T$ and $N$ and the conditions (H1) and (H2), we only prove (b) here. 
For $n \in \mathbb{N}$ and $t \in(0,1)$, we have from (H1) and (H2) that

$$
\begin{aligned}
\left|z_{n}^{\prime}(t)\right|= & \left|(1-t)\left((T N) x_{n}\right)^{\prime}(t)-t\left((T N) x_{n}\right)^{\prime}(t)+t(1-t)\left((T N) x_{n}\right)^{\prime \prime}(t)\right| \\
\leqslant & (1-t)\left|\left((T N) x_{n}\right)^{\prime}(t)\right|+t\left|\left((T N) x_{n}\right)^{\prime}(t)\right|+t(1-t)\left|\left((T N) x_{n}\right)^{\prime \prime}(t)\right| \\
\leqslant & (1-t) \int_{0}^{t} s\left|N x_{n}(s)\right| d s+(1-t) \int_{t}^{1}(1-s)\left|N x_{n}(s)\right| d s+(1-t)|D| \\
& \quad+t \int_{0}^{t} s\left|N x_{n}(s)\right| d s+t \int_{t}^{1}(1-s)\left|N x_{n}(s)\right| d s+t|D|+t(1-t)\left|N x_{n}(t)\right| \\
& \quad(1-t) \int_{0}^{t} s \chi(s) d s+(1-t) \int_{t}^{1}(1-s) \chi(s) d s+(1-t)|D| \\
& \quad+t \int_{0}^{t} s \chi(s) d s+t \int_{t}^{1}(1-s) \chi(s) d s+t|D|+t(1-t) \chi(t) \\
:= & \psi_{1}(t) .
\end{aligned}
$$

By (i) of Lemma 2.1,

$$
\psi_{1} \in L^{1}[0,1]
$$

Now (2.23) is sufficient to ensure the validity of (b) since

$$
\left|z_{n}\left(t_{1}\right)-z_{n}\left(t_{2}\right)\right|=\left|\int_{t_{2}}^{t_{1}} z_{n}^{\prime}(\tau) d \tau\right| \leqslant \int_{t_{2}}^{t_{1}}\left|z_{n}^{\prime}(\tau)\right| d \tau \leqslant \int_{t_{2}}^{t_{1}} \psi_{1}(\tau) d \tau .
$$

\section{Main RESULT}

TheOREM 3.1. Let $f:[0,1] \times \mathbb{R}^{2} \rightarrow \mathbb{R}$ satisfy the Carathéodory conditions. Assume that (H0),(H1) and (H2) hold. Then problem (1.4)-(1.5) has at least one solution in $X$ provided

$$
\|p\|_{E}\left(1+\frac{\sum_{i=1}^{m-2}\left|a_{i}\right|}{\left|1-\sum_{i=1}^{m-2} a_{i} \xi_{i}\right|}\right)+\|q\|_{L^{1}}<1
$$

REMARK 3.1. In [4], a key condition is that all $a_{i}$ have same sign. We don't need the restriction on $a_{i}$ in ( $\left.\mathrm{H} 0\right)$.

REMARK 3.2. Let us consider the three-point boundary value problem

$$
\begin{aligned}
x^{\prime \prime} & =g\left(t, x, x^{\prime}\right) \\
x^{\prime}(0) & =0, \quad x(1)=x\left(\frac{1}{3}\right)-x\left(\frac{2}{3}\right)
\end{aligned}
$$

where

$$
g(t, u, v)=\frac{\alpha}{t(1-t)} \sin (u+v) u+\beta v+\frac{1}{t(1-t)}\left[1+\cos \left(u^{200}+v^{30}\right)\right]
$$


It is easy to see that

$$
|g(t, u, v)| \leqslant p(t)|u|+q(t)|v|+r(t)
$$

with $p(t)=\alpha /(t(1-t)), q(t)=\beta$ and $r(t)=2 /(t(1-t))$. Clearly, $\|p\|_{E}=|\alpha|,\|q\|_{L^{1}}$ $=|\beta|,\|r\|_{E}=2$, and

$$
\frac{\sum_{i=1}^{m-2}\left|a_{i}\right|}{\left|1-\sum_{i=1}^{m-2} a_{i}\right|}=\frac{1+1}{|1-(1 \times(1 / 3)-1 \times(2 / 3))|}=\frac{3}{2}
$$

By Theorem 3.1, (3.2) has at least solution in $X$ provided

$$
\frac{5}{2}|\alpha|+|\beta|<1
$$

Now we cannot apply the main results of $[4]$ to deal with $(3.2)$ since $p, r \notin L^{1}[0,1]$.

Proof of Theorem 3.1. From Lemmas 2.2 and 2.3 , we know that $u \in X$ is a solution of (1.4)-(1.5) if and only if

$$
u=T N u \text {. }
$$

By Lemma 2.4, we can apply the Leray-Schauder continuation theorem (see, for example, [6, Corollary IV. 7]) to obtain the existence of a solution for (3.3) in $X$.

To do this it is suffices to verify that the set of all possible solutions of the family of equations

$$
\begin{aligned}
& x^{\prime \prime}(t)=\lambda f\left(t, x(t), x^{\prime}(t)\right)+\lambda e(t), \quad t \in(0,1) \\
& x(0)=0, \quad x(1)=\sum_{i=1}^{m-2} a_{i} x\left(\xi_{i}\right)
\end{aligned}
$$

is a priori bounded in $X$ by a constant independent of $\lambda \in[0,1]$.

Let $u \in X$ be a solution of $\left(3.4_{\lambda}\right)-(3.5)$ for some $\lambda \in[0,1]$. Then for $t \in[0,1]$, we have

$$
\begin{aligned}
|u(t)| & =\left|\int_{0}^{1} G(t, s) \lambda(N u)(s) d s+\frac{t}{1-\sum_{i=1}^{m-2} a_{i} \xi_{i}} \sum_{i=1}^{m-2} a_{i} \int_{0}^{1} G\left(\xi_{i}, s\right) \lambda(N u)(s) d s\right| \\
& =\left|\int_{0}^{1} G(t, s) u^{\prime \prime}(s) d s+\frac{t}{1-\sum_{i=1}^{m-2} a_{i} \xi_{i}} \sum_{i=1}^{m-2} a_{i} \int_{0}^{1} G\left(\xi_{i}, s\right) u^{\prime \prime}(s) d s\right| \\
& \leqslant\left(1+\frac{\sum_{i=1}^{m-2}\left|a_{i}\right|}{\left|1-\sum_{i=1}^{m-2} a_{i} \xi_{i}\right|}\right) \int_{0}^{1} s(1-s)\left|u^{\prime \prime}(s)\right| d s \\
& =\left(1+\frac{\sum_{i=1}^{m-2}\left|a_{i}\right|}{\left|1-\sum_{i=1}^{m-2} a_{i} \xi_{i}\right|}\right)\left\|u^{\prime \prime}(s)\right\|_{E}
\end{aligned}
$$

which implies that

$$
\|u\|_{\infty} \leqslant\left(1+\frac{\sum_{i=1}^{m-2}\left|a_{i}\right|}{\left|1-\sum_{i=1}^{m-2} a_{i} \xi_{i}\right|}\right)\left\|u^{\prime \prime}\right\|_{E}
$$


Similarly,

$$
\begin{aligned}
\left|t(1-t) u^{\prime}(t)\right| & =\left|t(1-t)\left[-\int_{0}^{t} s(\lambda N u)(s) d s+\int_{t}^{1}(1-s)(\lambda N u)(s) d s\right]\right| \\
& =\left|t(1-t)\left[-\int_{0}^{t} s u^{\prime \prime}(s) d s+\int_{t}^{1}(1-s) u^{\prime \prime}(s) d s\right]\right| \\
& \leqslant t(1-t) \int_{0}^{t} s\left|u^{\prime \prime}(s)\right| d s+t(1-t) \int_{t}^{1}(1-s)\left|u^{\prime \prime}(s)\right| d s \\
& \leqslant(1-t) \int_{0}^{t} s\left|u^{\prime \prime}(s)\right| d s+t \int_{t}^{1}(1-s)\left|u^{\prime \prime}(s)\right| d s \\
& \leqslant \int_{0}^{1} s(1-s)\left|u^{\prime \prime}(s)\right| d s
\end{aligned}
$$

which implies that

$$
\left\|t(1-t) u^{\prime}(t)\right\|_{\infty} \leqslant\left\|u^{\prime \prime}\right\|_{E}
$$

Now from (3.4), (3.7) and (3.9) it follows that

$$
\begin{aligned}
\left|t(1-t) u^{\prime \prime}(t)\right| & =\lambda t(1-t)\left|f\left(t, u(t), u^{\prime}(t)\right)+e(t)\right| \\
& \leqslant t(1-t)\left[p(t)|u(t)|+q(t)\left|u^{\prime}(t)\right|+|r(t)|+|e(t)|\right] \\
& \leqslant\|p\|_{E}\|u\|_{\infty}+\|q\|_{L^{1}}\left\|t(1-t) u^{\prime}(t)\right\|_{\infty}+\|r\|_{E}+\|e\|_{E} \\
& \leqslant\|p\|_{E}\left(1+\frac{\sum_{i=1}^{m-2}\left|a_{i}\right|}{\left|1-\sum_{i=1}^{m-2} a_{i} \xi_{i}\right|}\right)\left\|u^{\prime \prime}\right\|_{E}+\|q\|_{L^{1}}\left\|u^{\prime \prime}\right\|_{E}+\|r\|_{E}+\|e\|_{E}
\end{aligned}
$$

for $t \in(0,1)$. Thus

$$
\left\|u^{\prime \prime}\right\|_{E} \leqslant\left[\|p\|_{E}\left(1+\frac{\sum_{i=1}^{m-2}\left|a_{i}\right|}{\left|1-\sum_{i=1}^{m-2} a_{i} \xi_{i}\right|}\right)+\|q\|_{L^{1}}\right]\left\|u^{\prime \prime}\right\|_{E}+\|r\|_{E}+\|e\|_{E}
$$

It follows from the assumption (3.1) that there is a constant $c$, independent of $\lambda \in[0,1]$, such that

$$
\left\|u^{\prime \prime}\right\|_{E} \leqslant c
$$

This together (3.7) implies that

$$
\|u\|_{\infty} \leqslant\left(1+\frac{\sum_{i=1}^{m-2}\left|a_{i}\right|}{\left|1-\sum_{i=1}^{m-2} a_{i} \xi_{i}\right|}\right) c .
$$

Similarly, (3.12) together with (3.9) imply that

$$
\left\|t(1-t) u^{\prime}(t)\right\|_{\infty} \leqslant c
$$

Therefore

$$
\|u\|_{X} \leqslant \max \left\{c,\left(1+\frac{\sum_{i=1}^{m-2}\left|a_{i}\right|}{\left|1-\sum_{i=1}^{m-2} a_{i} \xi_{i}\right|}\right) c\right\} .
$$

This completes the proof of the theorem. 


\section{REFERENCES}

[1] R.P. Agarwal and D. O'Regan, 'Some new results for singular problems with sign changing nonlinearities', J. Comput. Appl. Math. 113 (2000), 1-15.

[2] H. Asakawa, 'Nonresonant singular two-point boundary value problems', Nonlinear Anal. 44 (2001), 791-809.

[3] J.V. Baxley, Some singular nonlinear boundary value problems, SIAM J. Math. Anal. 22 (1991), 463-469.

[4] C.P. Gupta, S.K. Ntouyas and P.Ch. Tsamatos, 'On an $m$-point boundary value problem for second order ordinary differential equations', Nonlinear Anal. 23 (1994), 1427-1436.

[5] R. Ma, 'Existence of positive solutions for superlinear semipositone $m$-point boundary-value problems', Proc. Edinburgh Math. Soc. (2) 46 (2003), 279-292.

[6] J. Mawhin, 'Topological degree methods in nonlinear boundary value problems', in NSF-CBMS Regional Conference Series in Mathematics 40 (Amer. Math. Soc., Providence, R.I., 1979).

[7] D. O'Regan, Theory of singular boundary value problems (World Scientific Publishing Co., Inc., River Edge, NJ, 1994).

[8] G. Shi and S. Chen, 'Positive solutions of fourth-order superlinear singular boundary value problems', Bull. Austral. Math. Soc. 66 (2002), 95-104.

[9] S.D. Taliaferro, 'A nonlinear singular boundary value problem', Nonlinear Anal. 3 (1979), 897-904.

[10] J.R.L. Webb Positive solutions of some three point boundary value problems via fixed point index theory, Nonlinear Anal. 47 (2001), 4319-4332.

[11] Z. Zhang and J. Wang, 'The upper and lower solution method for a class of singular nonlinear second order three-point boundary value problems', J. Comput. Appl. Math. $147(2002), 41-52$.

Department of Mathematics

Northwest Normal University

Lanzhou 730070

Peoples Republic of China
Department of Mathematics The University of Queensland

Brisbane Qld 4072

Australia 